\title{
E-Consent - a guide to implement an innovative solution to maintain recruitment in clinical trials during the COVID-19 pandemic
}

Ricardo Almeida-Magana ( $\square$ r.magana@ucl.ac.uk)

University College London Division of Surgery and Interventional Science https://orcid.org/0000-00023437-1649

Hanna Maroof

University College London Hospitals NHS Foundation Trust

Jack Grierson

University College London

Rosie Clow

University College London

\section{Eoin Dinneen}

University College London Hospitals NHS Foundation Trust

\section{Tarek Al-Hammouri}

University College London Hospitals NHS Foundation Trust

Nicola Muirhead

University College London Division of Medicine

Chris Brew-Graves

University College London Division of Medicine

John Kelly

University College London Division of Surgery and Interventional Science

\section{Greg Shaw}

University College London Hospitals NHS Foundation Trust

\section{Research Article}

Keywords: e-Consent, Consent management, prostate-cancer, informed consent

Posted Date: February 7th, 2022

DOI: https://doi.org/10.21203/rs.3.rs-1179173/v1

License: (a) (i) This work is licensed under a Creative Commons Attribution 4.0 International License. Read Full License 


\section{Abstract}

\section{Background:}

The COVID-19 pandemic has posed daunting challenges for the conduction of clinical research. Adopting new technologies such as remote electronic consent (e-Consent) can help overcome them. However, guidelines for e-Consent implementation in ongoing clinical trials are currently lacking. The NeuroSAFE PROOF trial is a randomised clinical trial evaluating the role of frozen section analysis during RARP for prostate cancer. In response to the COVID-19 crisis, recruitment was halted, and a remote e-Consent solution was designed. The aim of this paper is to describe the process of implementation, impact on recruitment rate and patients' experience using e-Consent.

\section{Methods:}

A substantial amendment of the protocol granted the creation of a remote e-Consent framework based on the REDCap environment, following the structure and content of the already approved paper consent form. A new pathway was developed which offered continuous support to patients through remote consultations. The whole process was judged to be compliant with regulatory requirements before implementation.

\section{Results:}

Before the first recruitment suspension, NeuroSAFE PROOF was recruiting an average of 9 patients per month. After e-Consent implementation, 63 new patients (4/month) have been enrolled despite a second lockdown, none of whom would have been recruited using the old methods given restrictions on face-toface consultations. Patients have given positive feedback on the use of the platform. Limited troubleshooting has been required after implementation.

\section{Conclusion:}

Remote e-Consent based recruitment was critical for the continuation of the NeuroSAFE PROOF trial during the COVID-19 pandemic. The described pathway complies with ethical and regulatory guidelines for informed consent, while minimizing face-to-face interactions that increase the risk of COVID-19 transmission. This guide will help researchers integrate e-Consent to ongoing or planned clinical trials while uncertainty about the course of the pandemic continues.

Trial registration: The NeuroSAFE PROOF trial has the registration number NCT03317990 (23 October 2017). Regional Ethics Committee; reference 17/LO/1978

\section{Introduction}

As of December 2021, over 265 million COVID-19 cases and more than 5 million associated deaths have been recorded worldwide [1]. The introduction of social distancing rules to curb the spread of the virus, 
staff redeployment to the intensive care unit, and prioritization of COVID-19 related research has not only resulted in a complete re-organization of hospital services but has also significantly impaired the conduction of clinical trials [20]. A study reviewing the ClinicalTrials.gov registry reported that 1052 trials were suspended, as a result of the pandemic, in 2020 [3]. Governing bodies such as the United Kingdom (UK) National Health Service (NHS) Health Research Authority and the Food and Drug Administration (FDA) have therefore issued guidelines to research teams on making appropriate changes to trial protocols $[4,5]$. The vast majority of these guidelines focus on a shift from on-site to remote conduction of study services.

However, obtaining patient consent poses a unique challenge, owing to the need for two-way communication between the research team and patient to ensure understanding of the provided information and subsequent documentation of approval in the form of a written signature. Previous literature has provided substantial evidence relating to the inadequacies of the traditional consent process [6]. Remote electronic consent (e-Consent) platforms have demonstrated distinct advantages over paper-based methods whilst also avoiding face-to-face interaction [7].

The NeuroSAFE PROOF trial is an ongoing, prospective, single-blinded, multi-centre, randomised controlled trial conducted on adult men undergoing robot-assisted radical prostatectomy (RARP) for nonmetastatic prostate cancer. Full trial description is publicly available [8]. The primary outcome is the difference in erectile function recovery between men undergoing standard RARP (control arm) and NeuroSAFE RARP (intervention arm) at 12 months following treatment. The trial enrolled its first patient in February 2019. However, recruitment was halted from March to June 2020 and from January to March 2021 due to the COVID-19 pandemic restrictions.

In response to the crisis and in line with the Health Research Authority (HRA) COVID-19 guidance released in May of 2020 [4], the NeuroSAFE PROOF research team decided to implement a Research Electronic Data Capture (REDCap) based e-Consent strategy. REDCap is a secure, web-based platform, with an integrated e-Consent feature set that was released in March of 2018 [9]. It allows for sharing of data within and across institutions, requires user authentication and can assign data access rights based on user role to maintain confidentiality [10].

During the development process, we noticed that guidelines surrounding the implementation of e-Consent to research protocols are currently lacking [11]. This paper provides guidance on how to implement this feature and reports on the impact these changes have made on our recruitment and patient consent process.

\section{Methods}

\section{Remote e-Consent instrument development:}

During the first recruitment pause, the University College London (UCL) Research Ethics Committee, Trial Management Group (TMG), Trial Steering Committee, and sponsor joined in a virtual meeting to decide 
the future of the trial. A substantial amendment of the protocol was approved that allowed the development of remote methods for screening, e-Consent, patient follow-up and data collection.

Our trial manager created a project for e-Consent within the REDCap environment, following the structure and content of the already approved paper consent form for NeuroSAFE Proof. The e-Consent framework works as a survey with a PDF Auto-Archiver feature. (Figure 1) Each question was added to the survey as a field with a yes/no format, except for identificatory and electronic signature fields. The latter allows the patient to sign the document using a mouse, stylus, or finger. This signature is captured and appended as a PNG image file with a timestamp.

During the enrolment process, if a patient provides an answer that disagrees with a consenting statement, the platform includes a hard stop feature, preventing ineligible patients from enrolling. The platform facilitates consent for visually impaired patients, allowing them to increase the font size as needed and features an integrated text-to-speech button.

In addition to the main trial e-Consent form, patients have the option to approve the use of the radical prostatectomy specimen obtained during surgery to be used for protocols approved by the UCL Biobanking program for future cancer research projects.

Owing to the process collecting patient identifiable data, the platform resides within the REDCap service being hosted behind the UCL Data Safe Haven [12], which conforms to NHS Data Security \& Protection Toolkit, General Data Protection Regulation, and ISO 27001 Information Security standards.

As much as possible, our team were determined to avoid missing data points. First, we instituted a system of postal patient-reported outcome measurements (PROM) collection in accordance with scheduled clinic visits. Second, we created an approved, automatic, online platform that allowed PROMs to be completed via email link and automatically embedded into the trial database.

\section{Patient E-consent pathway:}

Once a patient has been identified as potentially eligible for the trial in an MDT, a remote web-based virtual consultation within the normal care pathway for treatment discussion is performed by clinical staff (Figure 2). If inclusion and exclusion criteria are met, they are approached by a member of the study staff and are sent via secure NHS email a Patient Information Sheet (PIS). Within a week, they are reapproached via remote consultation. During this consultation the patient is encouraged to ask any question while the research staff can validate patients' understanding of the information.

If they agree, a unique e-Consent link is sent to their emails. The patient is then able to open the link on any personal electronic device and within a secure environment able to fill in the form. No time pressures allow the patient to read the consent form and a contact email is available to ask questions to the research team. 
After the patient answers all questions, a certification page is added to the document, displaying a copy of the responses, allowing the patient to confirm that all the information provided is correct before final submission. Subsequently, the responses are locked. This allows an authorized member of research staff to review the file and electronically co/sign and lock the document once again. The patient is sent an electronic copy, a copy is uploaded to their electronic medical file and a hard copy is printed and stored at the investigator site file within a locked and secure cabinet. Finally, patients who had consented and met all inclusion and exclusion criteria are randomised to either the control or intervention arm of the study.

\section{Results}

At the time of institutional lockdown in the UK, NeuroSAFE PROOF had 140 men 'on study' at four UK sites requiring ongoing oncological surveillance or care. As per HRA governmental advice, recruitment was suspended, and all face-to-face appointments were cancelled indefinitely. The trial was placed on hold for 12 weeks. Before this pause, the trial was recruiting an average of 9 patients per month, with an increasing trend, higher than the rate required to finish the study within the proposed timeline. (Figure 3 )

After implementing the remote consent and PROM collection, the NeuroSAFE PROOF study has subsequently been able to restart recruitment. We have been able to recruit 63 patients ( $4 / \mathrm{month}$ ) from the main site despite a second and third lockdown. Patient acceptance to the consent process has been exceptional, only 3 patients have requested the use of a paper consent form, all three of them citing a lack of familiarity with electronic devices as the main reason for the request.

Although we have not yet returned to pre-pandemic recruitment levels, as secondary sites have just restarted normal activities, the success of our online consent system means that we can expect to reach the planned sample size of 404 patients before December 2022.

Changing the follow-up modality for postal collection of PROMs and virtual visits have allowed us to complete 188 remote outpatient follow-up consultations, with only $6 \%$ of primary outcome data missing. These results would have been completely lost if this revolutionary package had not been introduced.

\section{Discussion}

There are three main aspects to informed consent. Firstly, to engage in a comprehensive discussion with the patient about the characteristics, goals, and procedures of the study with an emphasis on voluntary participation. Secondly, to allow the patient to use this information to make an informed decision and finally, to ensure the patients' decision is accurately documented [13]. These objectives are all addressed via the remote web-based methods that we have detailed in this paper. At the time of writing, this was the first study to describe the use of remote e-Consent in prostate cancer research. Our data demonstrate a significant uptake in patient recruitment after remote e-Consent implementation, which has allowed the continuation of our trial while complying with the government guidelines and simultaneously minimizing infection risk for patients and researchers. 
Early in the pandemic, a survey revealed that only $14 \%$ of oncology-focused European research institutions continued to enrol, with the focus being critical interventions for cancer patients [14]. Institutions were forced to implement criteria to decide which trials could continue, for example, Marcum et. al. reported that 29 of 130 active trials were cancelled at their centre [15]. A similar fate was expected to befall our trial. However, this was prevented by modification of the protocol via the described changes. Importantly, NeuroSAFE PROOF was the first trial focused on oncological surgery to reopen recruitment after a three-month halt during the first UK lockdown. Full trial protocol will be published according to the CONSERVE recommendations [16].

Furthermore, cancer research budgets within the UK have been drastically cut over the last two years, as charities have struggled to fundraise, and government budgets have been diverted [17]. Strategies such as E-consent can decrease the costs associated with clinical trials and may be key when determining funding allocation in the upcoming years. This was the case for our study when trial personnel were redeployed to frontline clinical services. However, due to the straightforward nature of the platform and lack of need for paperwork, one person can consent multiple patients and simultaneously monitor their responses in real-time. The trial was therefore able to continue with minimal staffing. Furthermore, no further costs to the project budget were incurred by the implementation of the platform as it used resources already available to the sponsor (UCL).

Our trial has been transformed from in-person to almost entirely remote conduction. Patients are now only required to travel to the hospital for surgery and post-operative catheter removal. E-consent, remote collection of PROMs, and virtual consultations have allowed for this shift to occur without the quality of clinical care or research standards being compromised. The uptake of technological platforms is particularly challenging in the older cohort of patients [18]. However, our transition to online consent was relatively seamless, indicating that REDCap is a user-friendly platform and therefore a strong alternative to paper consent forms, particularly for those shielding during the pandemic.

Before the COVID-19 crisis, most e-Consent platforms relied on the use of an in-clinic electronic device [11] Haussen et. al described the process of setting up an e-Consent platform using smartphones to communicate with legal representatives to authorize treatment for patients suffering from acute stroke [19]. This process was favoured amongst the representatives and even shortened trial enrolment time in comparison to paper consent methods. The smartphones provided adequate internet connection and trial personnel were readily available to resolve any troubleshooting issues [20]. However, face-to-face interaction poses an increased risk of COVID-19 transmission [21] and requires setting aside time for device disinfection in between patients [22]. Not only does our platform eliminate the risk of COVID-19 transmission but also allows patients to digest provided information in their own time. Patients are then able to utilize telehealth platforms to communicate any questions or concerns surrounding the consent form, directly with the research team. In addition, such platforms will potentially broaden the patient demographic of research trials such as NeuroSAFE [23]. Patients who may not have the means to travel back and forth to the clinic, who are unable to drive due to disability or who have caregiver responsibilities, will all be able to utilize this platform to provide informed consent without leaving the 
confines of their homes. REDCap based e-Consent utilizes these advantageous factors in confluence to overcome a multitude of challenges encountered with paper consent and as a result, may lead to a definitive switch from paper-based to online consent forms in the UK, irrespective of the COVID-19 pandemic. (Table 1)

Table 1

-Advantages and disadvantages of e-Consent

\begin{tabular}{|ll|}
\hline Advantages & Disadvantages \\
\hline Avoids physical attendance & Patients may be unfamiliar with electronic device use \\
\hline Requires fewer human resources & Privacy concerns if not properly set-up \\
\hline Requires less physical space & $\begin{array}{l}\text { Could introduce selection bias towards younger patients and } \\
\text { those with higher education }\end{array}$ \\
$\begin{array}{l}\text { Can be deployed to any number of } \\
\text { devices }\end{array}$ & $\begin{array}{l}\text { May decrease equitable access to trials across the } \\
\text { socioeconomic spectrum }\end{array}$ \\
\hline $\begin{array}{l}\text { Allows patients to answer in a } \\
\text { safe space }\end{array}$ & $\begin{array}{l}\text { Relies on patient access to electronic devices, email, and } \\
\text { internet connection }\end{array}$ \\
\hline $\begin{array}{l}\text { Can adapt to patient specific } \\
\text { disabilities }\end{array}$ & \\
\hline $\begin{array}{l}\text { Scalable } \\
\text { Reduces travel associated costs }\end{array}$ & \\
\hline $\begin{array}{l}\text { Reduces risk of contagion of } \\
\text { infectious diseases }\end{array}$ & \\
\hline $\begin{array}{l}\text { Integrated hard stops prevents } \\
\text { missing fields }\end{array}$ & \\
\hline Increases traceability & \\
\hline Removes postage cost & \\
\hline $\begin{array}{l}\text { Removes possibility of } \\
\text { transcription errors }\end{array}$ & \\
\hline
\end{tabular}

Other e-Consent platforms have demonstrated a vast array of additional advantages that can be utilized to optimize clinical trial recruitment rates. An example is the Research Permissions Management System (RPMS), developed in South Carolina [24]. This tool not only simplified the patient recruitment process but also matched patient to trials most tailored to them. Part of the RPMS consent was a section that allowed patients to opt-in to being contacted for future research. The ability to swiftly track patients who are willing to participate in clinical trials, without having to search through paper records, will inevitably facilitate the recruitment process for future studies. 
A study by Naeim et al has also shown video-based remote e-Consent to be useful in the obtainment of consent for biospecimen collection [25]. Patients are often uneasy about the relinquishment of bodily fluids and tissue samples for research. The reasons for this may include a lack of understanding of the research process or a lack of knowledge regarding where samples will be stored. Given the importance of biospecimen studies in answering current and future translational research questions, we utilized this knowledge to incorporate an optional separate section for biospecimen collection consent within our platform, to which patients generally agreed. Lastly, physicians and researchers have also used e-Consent to gain permission to access electronic health records for both clinical care and data-sharing between institutions [26]. This will enhance collaborative research and has the advantage of being a dynamic process, in which patients can easily opt-out or request re-consent.

There are several limitations to our study. Patients may have mixed preferences towards e-Consent [27]. Elderly patients are less familiar with the use of electronic devices and tend to be more sceptical about the trustworthiness of electronic records [28]. Since the average age of our cohort is younger than in other prostate cancer studies, owing to our inclusion criteria, we acknowledge that this could be one of the reasons for our success in using this platform. In addition, our cohort was exclusively male. There is no current research to indicate whether there is a difference in gender preferences towards online consent methods. The best approach for future studies would be to integrate both remote and in-person consent of female and male patients, maximizing opportunities to observe differences in outcomes amongst patients with different backgrounds.

\section{Conclusions}

The COVID-19 pandemic has completely changed the way clinical research is conducted and planned. Researchers must now consider social distancing restrictions while designing a protocol without compromising quality or patient safety. The implementation of a remote e-Consent pathway via REDCap was instrumental for the continuation of the NeuroSAFE PROOF trial throughout the pandemic. Both patients and regulatory bodies have accepted the use of this platform seamlessly and we have taken full advantage of the benefits of e-Consent. We hope the tools detailed in this paper will help researchers around the world make the necessary changes to ongoing studies or plan new proposals during these unprecedented times.

\section{Abbreviations}

e-Consent

Electronic consent

FDA

Food and Drug Administration

HRA

Health Research Authority

$\mathrm{NHS}$ 
National Health Service

PIS

Patient Information Sheet

PNG

Portable Network Graphics

PROM

Patient Reported Outcome Measurements

RARP

Robot Assisted Radical Prostatectomy

REDCap

Research Electronic Data Capture

RPMS

Research Permissions Management System

UCL

University College London

UK

United Kingdom

\section{Declarations}

\section{Ethics approval and consent to participate:}

Ethical approval for NeuroSAFE PROOF protocol version 5.0 was granted on 16 June 2021 REC reference 17/LO/1978). Written, informed consent to participate is obtained from all patients.

\section{Consent for publication:}

Not applicable

\section{Availability of data and materials:}

Data sharing is not applicable to this article as no datasets were generated or analysed during the current study. The NeuroSAFE PROOF trial is ongoing, and results will be published at study completion.

\section{Competing interests:}

The authors do not have any competing interests to declare.

\section{Funding:}

The NeuroSAFE PROOF is funded by the University College London Hospitals NHS Foundation Trust, The Rosetrees Foundation, the NIHR RfPB stream (reference: PB-PG-1216-200113), St Peter's Charitable Trust, and the Jon Moulton Charity Trust (charity no. 1109891) 


\section{Authors' contributions:}

- Study concept and design: GS, ED, JK, JG, RA, CBG, NM, RC

- Creation of the platform and pathway: ED, JG, RC

- Drafting of manuscript: HM, RA, JG, ED, RC, GS, TA, NM, CBG

- Critical revision of the manuscript for important intellectual content: All authors.

- Supervision: GS, JK, CBG, NM

\section{Acknowledgements:}

The authors would like to thank the following funding bodies who have provided the NeuroSAFE PROOF trial with funds to undertake this research: J P Moulton Foundation and the St. Peters Trust, the grant UL1 TR000445 from NCATS/NIH that allows REDCap to be freely available to non-profit institutions. The authors would also like to thank the IT for the School of Life and Medical Sciences Division and REDCap Data services in the Data Safe Haven team for their advice and support on information governance processes.

\section{Authors' information:}

N/A: No additional authors' information is required.

\section{References}

1. World Health Organization. WHO Coronavirus (COVID-19) Dashboard. https://covid19.who.int/. Accessed 3 December 2021.

2. Wyatt $D$, Faulkner-Gurstein $\mathrm{R}$, Cowan $\mathrm{H}$, Wolfe CDA. Impacts of COVID-19 on clinical research in the UK: A multi-method qualitative case study. PLOS ONE. 2021;16(8):e0256871.

3. Asaad M, Habibullah NK, Butler CE. The Impact of COVID-19 on Clinical Trials. Ann Surg. 2020;272(3):e222-e3.

4. NHS Health Research Authority. Making changes to a research study to manage the impact of COVID-19. https://www.hra.nhs.uk/covid-19-research/covid-19-guidance-sponsors-sites-andresearchers/ Accessed 3 December 2021.

5. FDA. Conduct of Clinical Trials of Medical Products During the COVID-19 Public Health Emergency. 2020. https://www.fda.gov/media/136238/download. Accessed 3 December 2021.

6. Nathe JM, Krakow EF. The Challenges of Informed Consent in High-Stakes, Randomized Oncology Trials: A Systematic Review. MDM Policy Practice. 2019;4(1):238146831984032.

7. Welch BM, Marshall E, Qanungo S, Aziz A, Laken M, Lenert L, et al. Teleconsent: A Novel Approach to Obtain Informed Consent for Research. Contemp Clin Trials Commun. 2016;3:74-9.

8. ClinicalTrials.gov. Evaluating the Effects of Frozen Section Technology on Oncological and Functional Outcomes at Radical Prostatectomy. (NeuroSAFEPROOF). 
https://clinicaltrials.gov/ct2/show/NCT03317990. Accessed 3 December 2021.

9. Harris PA, Taylor R, Thielke R, Payne J, Gonzalez N, Conde JG. Research electronic data capture (REDCap)-A metadata-driven methodology and workflow process for providing translational research informatics support. J Biomed Inform. 2009;42(2):377-81.

10. Lawrence CE, Dunkel L, Mcever M, Israel T, Taylor R, Chiriboga G, et al. A REDCap-based model for electronic consent (eConsent): Moving toward a more personalized consent. Journal of Clinical Translational Science. 2020;4(4):345-53.

11. Lunt $\mathrm{H}$, Connor $\mathrm{S}$, Skinner $\mathrm{H}$, Brogden $\mathrm{G}$. Electronic informed consent: the need to redesign the consent process for the digital age. Internal Medicine Journal. 2019;49(7):923-9.

12. University College London. Data Safe Haven (DSH). https://www.ucl.ac.uk/isd/services/file-storagesharing/data-safe-haven-dsh. Accessed 3 December 2021.

13. Grady C, Cummings SR, Rowbotham MC, Mcconnell MV, Ashley EA, Kang G. Informed Consent. N Engl J Med. 2017;376(9):856-67.

14. Upadhaya S, Yu JX, Oliva C, Hooton M, Hodge J, Hubbard-Lucey VM. Impact of COVID-19 on oncology clinical trials. Nat Rev Drug Discov. 2020;19(6):376-7.

15. Marcum M, Kurtzweil N, Vollmer C, Schmid L, Vollmer A, Kastl A, et al. COVID-19 pandemic and impact on cancer clinical trials: An academic medical center perspective. Cancer Med. 2020;9(17):6141-6.

16. Orkin AM, Gill PJ, Ghersi D, Campbell L, Sugarman J, Emsley R, et al. Guidelines for Reporting Trial Protocols and Completed Trials Modified Due to the COVID-19 Pandemic and Other Extenuating Circumstances. JAMA. 2021;326(3):257.

17. Burki TK. Cuts in cancer research funding due to COVID-19. The Lancet Oncology. 2021;22(1):e6.

18. Wilson J, Heinsch M, Betts D, Booth D, Kay-Lambkin F. Barriers and facilitators to the use of e-health by older adults: a scoping review. BMC Public Health. 2021;21(1).

19. Haussen DC, Doppelheuer S, Schindler K, Grossberg JA, Bouslama M, Schultz M, et al. Utilization of a Smartphone Platform for Electronic Informed Consent in Acute Stroke Trials. Stroke. 2017;48(11):3156-60.

20. Haussen DC, Craft L, Doppelheuer S, Rodrigues GM, Al-Bayati AR, Ravindran K, et al. Legal authorized representative experience with smartphone-based electronic informed consent in an acute stroke trial. Journal of Neurolnterventional Surgery. 2020;12(5):483.

21. Meyerowitz EA, Richterman A, Gandhi RT, Sax PE. Transmission of SARS-CoV-2: A Review of Viral, Host, and Environmental Factors. Ann Intern Med. 2021;174(1):69-79.

22. Ong SWX, Tan YK, Chia PY, Lee TH, Ng OT, Wong MSY, et al. Air, Surface Environmental, and Personal Protective Equipment Contamination by Severe Acute Respiratory Syndrome Coronavirus 2 (SARSCoV-2) From a Symptomatic Patient. JAMA. 2020;323(16):1610.

23. De Sutter E, Borry P, Geerts D, Huys I. Personalized and long-term electronic informed consent in clinical research: stakeholder views. BMC Medical Ethics. 2021;22(1). 
24. Sanderson IC, Obeid JS, Madathil KC, Gerken K, Fryar K, Rugg D, et al. Managing clinical research permissions electronically: A novel approach to enhancing recruitment and managing consents. Clin Trails. 2013;10(4):604-11.

25. Naeim A, Dry S, Elashoff D, Xie Z, Petruse A, Magyar C, et al. Electronic Video Consent to Power Precision Health Research: A Pilot Cohort Study. JMIR Formative Research. 2021;5(9):e29123.

26. Heinze 0 , Birkle M, Köster L, Bergh B. Architecture of a consent management suite and integration into IHE-based regional health information networks. BMC Med Inform Decis Mak. 2011;11(1):58.

27. Harle CA, Golembiewski EH, Rahmanian KP, Krieger JL, Hagmajer D, Mainous AG, et al. Patient preferences toward an interactive e-consent application for research using electronic health records. J Am Med Inform Assoc. 2018;25(3):360-8.

28. Simon CM, Schartz HA, Rosenthal GE, Eisenstein EL, Klein DW. Perspectives on Electronic Informed Consent From Patients Underrepresented in Research in the United States: A Focus Group Study. Journal of Empirical Research on Human Research Ethics. 2018;13(4):338-48.

\section{Figures}

\section{Figure 1}

Example of a patients view of the e-Consent questionnare.

\section{Figure 2}

E-consent pathway diagram.

\section{Figure 3}

Rate of recruitment of the NeuroSAFE trial and ideal recruitment rate. 